\title{
A Multi-stage Approach for 3D Teeth Segmentation from Dentition Surfaces ${ }^{\star}$
}

\author{
Marcin Grzegorzek ${ }^{1}$, Marina Trierscheid ${ }^{1,2, \star \star}$, \\ Dimitri Papoutsis ${ }^{2}$, and Dietrich Paulus ${ }^{1}$ \\ 1 Research Group for Active Vision, University of Koblenz-Landau \\ Universitätsstr. 1, D-56070 Koblenz \\ http://www.uni-koblenz.de/agas \\ 2 RV realtime visions GmbH, Technology Center Koblenz \\ Universitätsstr. 3, D-56070 Koblenz \\ http://www.realtimevisions.com
}

\begin{abstract}
In this paper, we present a multi-stage approach for teeth segmentation from 3D dentition surfaces based on a 2D model-based contour retrieval algorithm. First, a 3D dentition model is loaded to the system and a range image is created. Second, binarized 2D sectional images are generated and contours are extracted. During several processing steps a set of tooth contour candidates are produced and they are evaluated. The best-fitting contour for each tooth is refined using snakes. Finally, the 2D contours are integrated to full 3D segmentation results. Due to its excellent experimental results, our algorithm has been applied in the practical realization of a so-called virtual articulator currently being developed for dentistry. Today, only mechanical articulators are applied in the dental practice. They are used in the fabrication and testing of removable prosthodontic appliances (dentures), fixed prosthodontic restorations (crowns, bridges, inlays and onlays), and orthodontic appliances. Virtual articulators are supposed to simulate the same functionality, however, in a much more flexible and convenient way.
\end{abstract}

\section{Introduction}

Dental treatments often require a functional analysis of movements of the mandible and of the various tooth-to-tooth relationships that accompany those movements. Currently this is practically done by a device called mechanical articulator. Mechanical articulators simulate the temporomandibular joints and jaws to which maxillary and mandibular casts are attached (see Figure 1, left). They are used in the fabrication and testing of removable prosthodontic appliances (dentures), fixed prosthodontic restorations (crowns, bridges, inlays and onlays), and orthodontic appliances. Mechanical articulators, however, feature

\footnotetext{
* Research activities leading to this work have been supported by the German Federal Ministry of Economics and Technology within the funding scheme PRO INNO II.

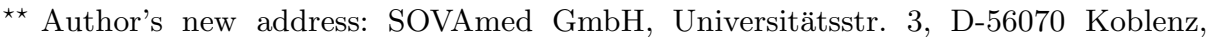
http://www. sovamed.com
}

A. Elmoataz et al. (Eds.): ICISP 2010, LNCS 6134, pp. 521-530, 2010.

(C) Springer-Verlag Berlin Heidelberg 2010 

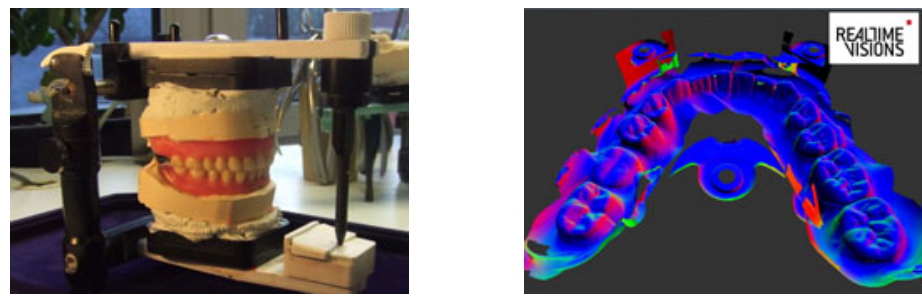

Fig. 1. Left: typical mechanical articulator. Right: 3D dentition model for the virtual articulator.

some limitations. They have a small number of degrees of freedom, and their spatial accuracy is rather moderate - at least in comparison to the precision of state-of-the-art 3D scanners nowadays available in the medical industry.

For these reasons, a project funded by the German Federal Ministry of Economics and Technology aiming at conceptualizing and developing a virtual articulator has been initialized. The virtual articulator is a system with the same practical application as the mechanical one, however, it works in a much more precise and convenient way. The system consists of three parts: an impression tray, a device for the analysis of jaw motion using ultrasonic sound sensors, and a high-precision optical 3D scanner for the digital acquisition of the dental impression. Thus the anatomical information is combined with the actual motion of the maxillary and mandibular teeth in relation to each other. One goal of the virtual articulator is to determine the teeth antagonists and the occlusion surfaces between them. This analysis can be accomplished without any limitations of degrees of freedom. However, apart from the teeth areas, neighbouring surfaces such as gums and teethridge are also included in the dentition models (see Figure 1, right). Therefore, the first and unavoidable step in designing a virtual articulator is the segmentation of teeth from the $3 \mathrm{D}$ dentition models.

The list of requirements for the teeth segmentation approach has been created by dental doctors and technicians involved in the project. The segmentation algorithm must: (i) work with a high geometrical accuracy, (ii) require little user interaction, and (iii) provide segmentation results for a jaw with 16 teeth within few seconds. These requirements have significantly influenced the selection of our segmentation strategy and serve as a basis for its evaluation. Our approach starts with generating $2 \mathrm{D}$ slices from the $3 \mathrm{D}$ dentition models given as range images. Thus, the problem is reduced to a $2 \mathrm{D}$ segmentation task. One can distinguish between four main categories of 2D image segmentation algorithms, namely the pixel-based, the region-based, the contour-based, and the model-based methods [1. The pixel-based and the region-based approaches work very fast and do not require any user interaction, however, due to their simplicity they are unable to overcome the problem of artefacts in the data (e.g., wholes in the 3D dentition models). The contour-based strategy, still fast and fully automatic, is suitable for segmenting structures with clear boundaries. Unfortunately, neighbouring tissue surfaces visualized in the dentition models are very similar to 
each other and there are no clear borders between them. Model-based methods are able to integrate a priori knowledge into the segmentation process which is their huge advantage, if the application domain is so clearly defined as in our case. Therefore, our own approach for the segmentation of teeth in 2D range images uses several model-based techniques, including active contours. Active contours adopt aspects of the contour-based and the region-based methods and integrate object-based model knowledge into the segmentation process. Finally, we summarize the $2 \mathrm{D}$ image segmentation results back to the $3 \mathrm{D}$ space which provides the $3 \mathrm{D}$ teeth models.

The paper is structured as follows. Section 2 overviews most relevant approaches for 3D segmentation. In Section 3, our own segmentation method is described. Section 4 comprehensively evaluates the approach using a manually created ground truth. Section 5 concludes the paper and states shortly our future work in the context of the virtual articulator.

\section{Related Work}

Automatic teeth segmentation algorithms can be used for various purposes and there is some related work in this area. However, these approaches do not altogether fulfil the project's requirements specified in section 11. Therefore, the segmentation algorithm used for the virtual articulator has been designed for that purpose in particular, which makes it original and novel.

The most related work has been published by Kondo et al. in [2]. This paper presents an automated method for tooth segmentation from 3D digitized image captures by a laser scanner. Similar to our approach, the authors avoid the complexity of directly processing $3 \mathrm{D}$ mesh data and process range images. They use two range images. First, they obtain the dental arch from the plan-view range image and then compute a panoramic range image of the dental model based on the dental arch. The interstices between the teeth are detected separately in the two range images, and results from both views are combined for a determination of interstice locations and orientations. Finally, the teeth are separated from the gums by delineating the gum margin. The authors tested their algorithm on 34 dental models. No gold standard has been used for the evaluation. Moreover, their dental models seem to be very clean and free from the artifacts which our models show.

There are further scientific contributions in this area. In [3] an automatic tooth segmentation method using active contours without edges is presented. Here, the authors concentrate on the identification of deceased individuals based on dental characteristics. However, in contrast to our approach, side views of the teeth are segmented in this work. Another related approach has been published in [4] where the authors present a multi-step approach based on geometric types of active contours for volumetric computed tomography (CT). Finally, Zhao et al. introduce an interactive method for teeth segmentation in [5]. Based on curvature values of the triangle mesh, feature points are connected to feature regions and a feature contour is obtained with the help of user supplied information. 


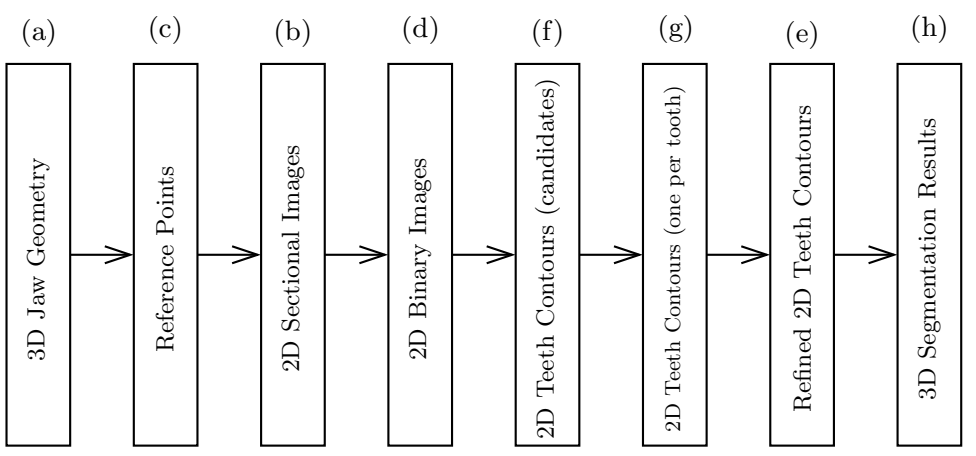

Fig. 2. Processing chain of our segmentation algorithm serving as a road map for Section 3

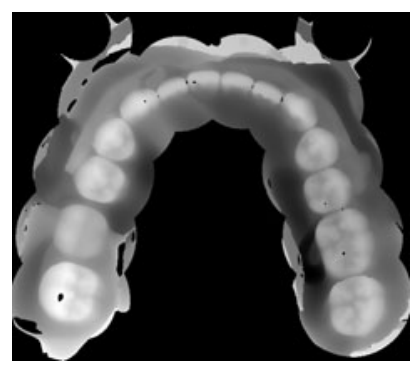

Fig. 3. Range image generated from a dentition model

\section{Segmentation Approach}

In this section, we describe our teeth segmentation algorithm. Its processing chain is depicted in Figure 2, where steps from (a) to (d) can be considered as preprocessing (Subsection 3.1) and steps from (e) to (h) contain the computation of teeth contours in $2 \mathrm{D}$ range images as well as their summary to $3 \mathrm{D}$ segmentation results (Subsection 3.2).

\subsection{Preprocessing}

First, a 3D dentition model is loaded to the system in form of a 3D jaw geometry (STL format) and a range image is created from it (Figure 3). Second, the user sets one reference point near the center of each tooth seen in the range image. Third, slices of the denture surface are cut on multiple planes and a range image is created from each slice, resulting in several $2 \mathrm{D}$ sectional images. Finally, the sectional images are binarized using a simple thresholding so that white pixels represent available geometry and black pixels depict the background. 

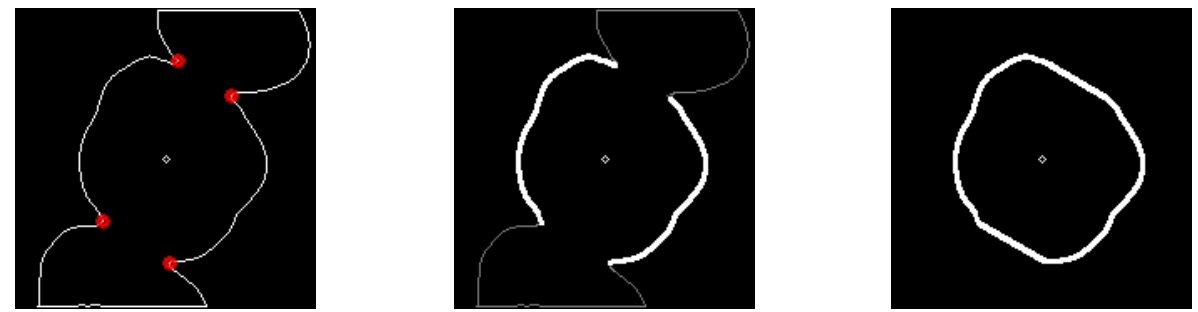

Fig. 4. Left: split points of the main contour. Middle: contour segments after splitting. Right: reshaped contour. The circle in the middle of the images marks the reference point.

\subsection{D Teeth Contours and 3D Segmentation Results}

For each reference point, and thus for each tooth, 2D contours are extracted from each binary images resulting from the preprocessing. Therefore in each binary image a square proximity region around the reference point with a fixed size is taken into consideration. The iterative contour extraction algorithm of Suzuki is applied for each region row by row [6]. As a result, all contours are stored in a tree data structure, the contour tree. Depending on the kind of each contour (white to black passage, or black to white passage), it is denoted either as internal or external. Obviously, internal contours represent artefacts (wholes) in the binary images (black pixels in a white region) and are disregarded from further investigations. The longest external contour of the contour tree which encloses the reference point is chosen to be the main contour for the following steps of the algorithm. The main contour resulting from a region around a molar looks as depicted in Figure 4 .

In order to reshape this contour so that it matches the tooth outlines, split points between the teeth (highlighted in Figure 4) must be determined. For this purpose, dominant points of the contour are detected first. Candidates $\boldsymbol{p}$ for dominant points are identified by inscribing triangles in the contour as in Figure 5. We used the algorithm of Chetverikov [7. Here, a dominant point fulfills the following criteria

$$
\begin{gathered}
d_{\min } \leq\left\|\boldsymbol{p}-\boldsymbol{p}^{+}\right\| \leq d_{\max }, d_{\min } \leq\left\|\boldsymbol{p}-\boldsymbol{p}^{-}\right\| \leq d_{\max } \\
\alpha \leq \alpha_{\max }
\end{gathered}
$$

$\left\|\boldsymbol{p}-\boldsymbol{p}^{+}\right\|=a$ and $\left\|\boldsymbol{p}-\boldsymbol{p}^{-}\right\|=b$ are Euclidean distances between the points. $\alpha \in[-\pi, \pi]$ is the opening angle at the point $\boldsymbol{p}$.

In addition to the dominant points, points on which the convexity property of the main contour is not fulfilled are also calculated. They are quantitatively expressed by a measure of nonconvexity. Those with a high value of this measure (greater than a particular threshold) are called convexity defects. The split points between the teeth are eventually identified by the intersection between the set of dominant points and the set of convex-

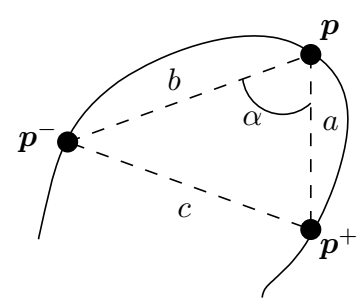

Fig. 5. Finding candidates for dominant points 
ity defects. Splitting up the main contour using these points results in several contour segments. Segments not matching the outline of the tooth are discarded using a priori knowledge about the characteristics of these outlines. Finally, the remaining contour segments (Figure 4, right) are used to create a candidate tooth contour for this tooth. They are connected to each other based on the Bresenham line algorithm.

Up to this point (step (e) in Figure 2), several candidates for the tooth contours have been retrieved. In the next step, for each tooth the best-fitting contour will be determined. Therefore we have defined certain criteria in order to evaluate the candidates. They are based on contour form features involving the following:

area $\boldsymbol{A}$ number of pixels enclosed by the contour

perimeter $\boldsymbol{P}$ length of the contour as the sum of distances of consecutive points compactness $c$ measure for the efficiency of the contour to contain a given area $A$, defined as

$$
c=\frac{P^{2}}{4 \pi A} .
$$

The compactness of a circle equals one, less compact contours result in a value greater than one.

Next, from all candidate contours three reference values are identified: the maximum area maxArea, the smallest measured value of compactness minCompactness and the smallest of all major convexity defects minConvexityDefect. The best-fitting contour for a certain tooth can now be retrieved by evaluating the following simple optimization vector $v$ for each candidate $i$ :

$$
\begin{gathered}
\boldsymbol{v}[1]=\frac{\text { contour }[i] \cdot \text { area }}{\text { maxArea }} \cdot \alpha \\
\boldsymbol{v}[2]=\frac{\text { minConvexityDefect }}{\text { contour }[i] \cdot \text { majorConvexityDefect }} \cdot \beta \\
\boldsymbol{v}[3]=\frac{\text { minCompactness }}{\text { contour }[i] . \text { compactness }} \cdot \gamma
\end{gathered}
$$

The factors $\alpha, \beta, \gamma$ are weights for the criteria. The vector having the greatest magnitude finally refers to the "optimal" tooth contour among the candidates.

A contour found by the algorithm described above usually encloses most of the tooth area, but the boundaries may be imprecise. This is due to retrieving the contours from sectional images which only represent a part of the dentition model. Therefore, the tooth contour is refined using the snakes algorithm on a gradient image computed from a range image of the model. A snake, a special case of active contours, is an energy-minimizing contour guided by external constraint forces and influenced by image forces that pull it toward features such as lines and edges [8]. The application of snakes results in a correction of the tooth contours toward the outlines of each tooth, as long as this outline is discriminable in the gradient image to a certain extend. 

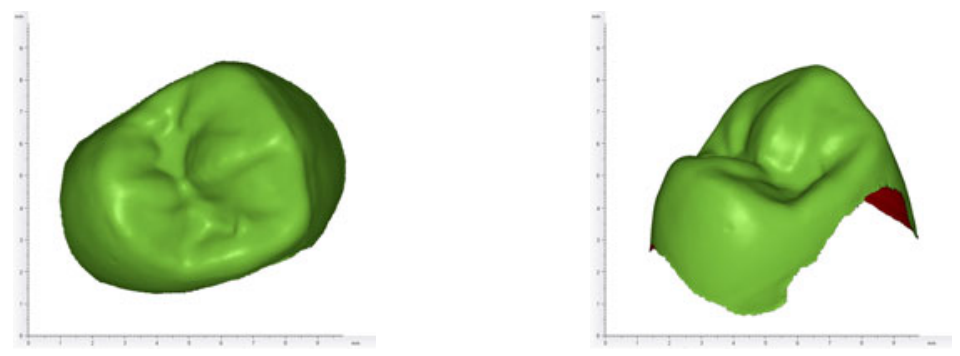

Fig. 6. An example 3D segmentation result for a premolar from two points of view

In the final step of our method, the $2 \mathrm{D}$ refined contours computed for a particular tooth are integrated into a complete 3D tooth segmentation result. If a single tooth of the dentition model is needed, the particular contour is taken into account and the subjacent 1 surface is extracted. The set of all teeth surfaces can be retrieved by extracting all surface regions which are enclosed by any tooth contour. An example segmentation result for a premolar visualized from two different points of view can be found in Figure 6 .

\section{Experiments and Results}

For experiments, we used altogether 28 pairs of $3 \mathrm{D}$ dentition models given in the STL format. They have been scanned via an optical 3D dental scanner. We divided them into three categories. The first one (dataset I) consists of 8 pairs acquired from artificial sets of teeth created by an experienced dental technician. It represents ideal teeth in the sense of its anatomical features (e. g. the characteristics of fissures and cusps). The second one (dataset II) includes 17 pairs of dental models which have been acquired from European patients with healthy teeth. Although the surfaces of these models are very smooth, some teeth feature malpositions, which makes the segmentation task more difficult. The third category (dataset III) consists of 4 pairs of dentition models. Here, the malposition of teeth is more significant. All teeth have been manually segmented by an experienced dental technician, which provided a gold standard for the evaluation.

In our experiments, we have distinguished between the four different kinds of human teeth, namely incisors, canines, premolars and molars 2 Apparently the difficulty of the segmentation task differs significantly from one teeth category to another, which is why we have evaluated them separately. The accuracy in the determination of the $2 \mathrm{D}$ tooth contour has been evaluated using well-known discrepancy measures: the sensitivity and specificity as well as the Tanimoto and Dice measures.

\footnotetext{
${ }^{1}$ considering the point of view from which the range image has been created before

${ }^{2}$ Incisors -1 st and 2 nd in the quadrant, canines $-3 \mathrm{rd}$, premolars - 4th and 5th, molars -6 th, 7 th and 8th.
} 
Table 1. Mean values of the sensitivity (Se), specificity (Sp), Tanimoto measure (Ta), and Dice measure (Di) achieved by our algorithm for teeth contour estimation calculated for four different types of teeth and three different datasets

\begin{tabular}{|c|c|c|c|c|c|c|c|c|c|c|c|c|c|c|c|c|}
\hline \multirow{2}{*}{$\begin{array}{c}\text { Data } \\
\text { Set }\end{array}$} & \multicolumn{4}{|c|}{ Incisors } & \multicolumn{4}{|c|}{ Caninus } & \multicolumn{4}{|c|}{ Premolars } & \multicolumn{4}{|c|}{ Molars } \\
\hline & Se & \begin{tabular}{|l|} 
Sp \\
\end{tabular} & Ta & Di & Se & \begin{tabular}{|l|}
$\mathbf{S p}$ \\
\end{tabular} & $\mathbf{T a}$ & $\overline{D i}$ & Se & Sp & Ta & \begin{tabular}{|l}
$\mathbf{D i}$ \\
\end{tabular} & Se & Sp & $\mathbf{T a}$ & Di \\
\hline $\bar{I}$ & 0.82 & 1.00 & 0.76 & 0.86 & 0.83 & 1.00 & 0.77 & 0.87 & 0.99 & 0.99 & 0.92 & 0.96 & 0.97 & 0.98 & 0.9 & 0.9 \\
\hline II & 0.86 & 0.99 & 0.79 & 0.87 & 0.90 & 0.99 & 0.84 & 0.91 & 0.99 & 0.98 & 0.91 & 0.95 & 0.95 & 0.96 & 0.85 & 0.92 \\
\hline III & 0.54 & 0.80 & 0.45 & 0.56 & 0.76 & 0.99 & 0.71 & 0.82 & 0.96 & 0.98 & 0.88 & 0.94 & 0.94 & 0.95 & 0.84 & 0.90 \\
\hline
\end{tabular}

Table 2. Standard deviations of the sensitivity (Se), specificity (Sp), Tanimoto measure (Ta), and Dice measure (Di) achieved by our algorithm for teeth contour estimation calculated for four different types of teeth and three different datasets

\begin{tabular}{|c||c|c|c|c||c|c|c|c||c|c|c|c||c|c|c|c|}
\hline \multicolumn{1}{|c|}{$\begin{array}{c}\text { Data } \\
\text { Set }\end{array}$} & \multicolumn{4}{|c||}{ Incisors } & \multicolumn{4}{c||}{ Caninus } & \multicolumn{3}{c||}{ Premolars } & \multicolumn{4}{c|}{ Molars } \\
\hline \hline I & Sp & Ta & Di & Se & Sp & Ta & Di & Se & Sp & Ta & Di & Se & Sp & Ta & Di \\
\hline II & 0.13 & 0.01 & 0.14 & 0.12 & 0.12 & 0.01 & 0.10 & 0.06 & 0.02 & 0.01 & 0.02 & 0.01 & 0.05 & 0.01 & 0.05 & 0.02 \\
\hline III & 0.33 & 0.38 & 0.26 & 0.07 & 0.11 & 0.01 & 0.09 & 0.06 & 0.02 & 0.01 & 0.04 & 0.02 & 0.14 & 0.04 & 0.13 & 0.10 \\
\hline
\end{tabular}

Let $A$ and $B$ be two compact sets of image elements of the sizes $|A|$ and $|B|$.

\section{Dice coefficient}

$$
C_{D}=\frac{2|A \cap B|}{|A|+|B|}
$$

\section{Tanimoto coefficient}

$$
C_{T}=\frac{|A \cap B|}{|A \cup B|}
$$

Let $N$ be the set of all image elements of the size $|N|$ and $A, B$ two compact sets of image elements of the sizes $|A|,|B|$, where $B$ is the gold standard.

Sensitivity of $A$

$$
S_{e}=\frac{|A \cap B|}{|B|}
$$

Specificity of $A$

$$
S_{p}=\frac{|N|-|A \cup B|}{|N|-|B|}
$$

The mean values of the achieved results can be found in Table 1 , the standard deviations in Table 2 .

As one can see, remarkable high performance has been achieved in all three datasets for premolars and molars (see example results in Figure 7). Incisors cause our algorithm difficulties, especially those of dataset III. 

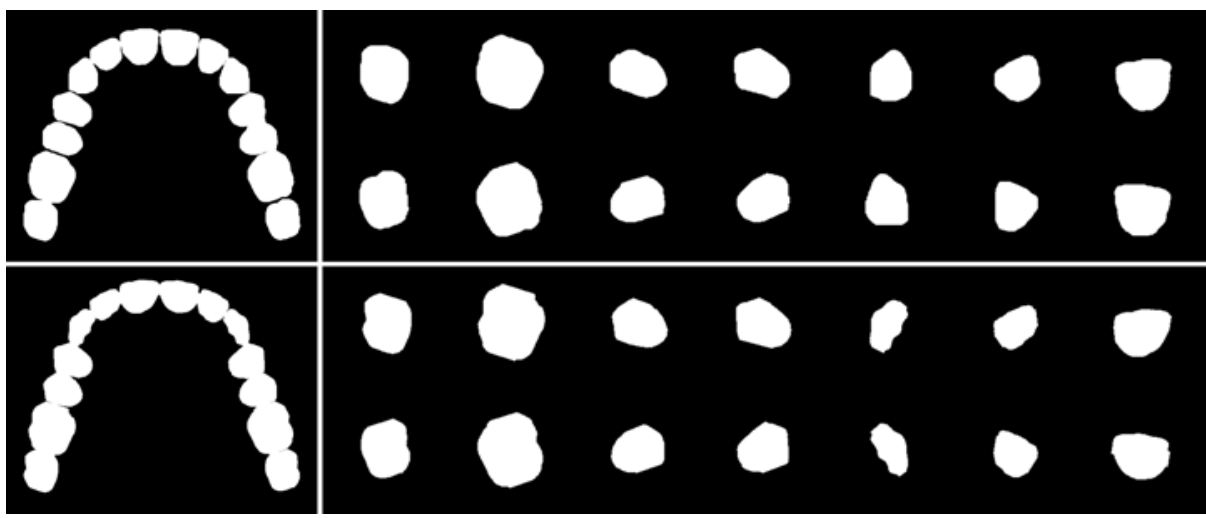

Fig. 7. Above: gold standard of an upper jaw of dataset I in the form of binary masks. Below: corresponding segmentation result.

\section{Conclusions}

In this work, we presented a new algorithm for the segmentation of teeth in $3 \mathrm{D}$ dentition models. The approach will be used in the practical realization of the so-called virtual articulator. The requirements for our method defined by a dental technician include a high geometrical accuracy, limited user interaction, and execution time efficiency. In the development and evaluation phase of our algorithm we focused on the $2 \mathrm{D}$ tooth contour determination which finally served as a basis for the full 3D tooth segmentation.

In the only interactive step of our approach, the user is required to set one reference point per teeth manually in a range image. This is necessary, since our initial experiments with fully automatic methods have not satisfied the industrial requirements. Usually, there are 14 to 16 teeth in a dentition model. Thus, the manual effort has been significantly reduced in comparison to a previous method, where the teeth contours had been segmented completely manually. The execution time of few seconds from setting the reference points until achieving the segmentation results for one dentition model also fulfills the requirements of the virtual articulator. The geometrical accuracy of our segmentation approach has been evaluated for more than 800 teeth against a gold standard created by an experienced dental technician. The high sensitivity and specificity achieved in our experiments, especially for premolars and molars, prove the applicability of our method in the practical realization of the virtual articulator.

In the future, we will analyse range images taken from frontal views of the teeth in order to increase the segmentation accuracy for incisors. 


\section{References}

1. Lehmann, T., Oberschelp, W., Pelikan, E., Repges, R.: Bildverarbeitung für die Medizin: Grundlagen, Modelle, Methoden, Anwendungen. Springer, Heidelberg (1997)

2. Kondo, T., Ong, S.H., Foong, K.W.C.: Tooth segmentation of dental study models using range images. IEEE Transactions on Medical Imaging 23(3), 350-362 (2004)

3. Shah, S., Abaza, A., Ross, A., Ammar, H.: Automatic tooth segmentation using active contour without edges. In: 2006 Biometrics Symposium, Baltimore, USA, August 2006, pp. 1-6. IEEE Computer Society, Los Alamitos (2006)

4. Keyhaninejad, S., Zoroofi, R.A., Setarehdan, S.K., Shirani, G.: Automated segmentation of teeth in multi-slice ct images. In: International Conference on Visual Information Engineering, Bangalore, India, September 2006, pp. 339-344. IEEE Computer Society, Los Alamitos (2006)

5. Zhao, M., Ma, L., Tan, W., Nie, D.: Interactive tooth segmentation of dental models. In: IEEE Conference on Engineering in Medicine and Biology, Shanghai, China, September 2005, pp. 654-657. IEEE Computer Society, Los Alamitos (2005)

6. Suzuki, S., Abe, K.: Topological structural analysis of digitized binary images by border following. Computer Vision, Graphics, and Image Processing 30(1), 32-46 (1985)

7. Chetverikov, D.: A simple and efficient algorithm for detection of high curvature points in planar curves. In: Petkov, N., Westenberg, M.A. (eds.) CAIP 2003. LNCS, vol. 2756, pp. 746-753. Springer, Heidelberg (2003)

8. Kass, M., Witkin, A., Terzopoulos, D.: Snakes: Active contour models. International Journal on Computer Vision 1(4), 321-331 (2004) 